\title{
Systemic Quinolones and Risk of Acute Liver Failure II: Systematic Review of Clinical Trials
}

\section{Mohamed Kadry Taher ${ }^{1,2,3^{*}}$ (i), Mohamed Habsah, ${ }^{2,4}$, Lise Bjerre ${ }^{2,5,6}$, Franco Momoli2,3,5, Donald Mattison $^{1,3}$ and Daniel Krewski, ${ }^{1,2,3}$}

${ }^{1}$ McLaughlin Centre for Population Health Risk Assessment, Faculty of Medicine, University of Ottawa, Canada

${ }^{2}$ School of Epidemiology and Public Health, University of Ottawa, Canada

${ }^{3}$ Risk Sciences International, Canada

${ }^{4}$ Canadian Red Cross, Canada

${ }^{5}$ Department of Family Medicine, University of Ottawa, Canada

${ }^{6}$ C.T. Lamont Primary Health Care Research Centre, Bruyère Research Institute, Canada

${ }^{7}$ Children's Hospital of Eastern Ontario Research Institute, Canada

*Corresponding author: Dr. Mohamed Kadry Taher, McLaughlin Centre for Population Health Risk Assessment, Faculty of Medicine, University of Ottawa, 600 Peter Morand Crescent, Room 216, Ottawa, ON, K1G 5Z3, Canada, Tel: +1-(613)562-5381, Fax: +1-(613)-562-5380

\begin{abstract}
Background: Quinolones are a class with four generations of synthetic antibiotics characterized by a unique mechanism of action, broad spectrum, potent pharmacologic properties and reasonable safety profile. Their global and growing popularity has been accompanied by an increase in the emergence of antimicrobial resistance and occurrence of unexpected adverse reactions. Nevertheless, physicians continue to prescribe these drugs on an increasing scale, irrespective of the availability of other treatment alternatives.

Objective: To systematically review all clinical trials where a quinolone antibiotic was tested or used as a comparator to other drugs or drug combinations, for evidence on quinolones' association with ALF risk.
\end{abstract}

Methods: We examined 4 major bibliographic databases, 8 clinical trial registries, and major grey literature sources including international conference proceedings, drug review networks and databases of pharmaceutical companies for ongoing or unpublished studies. We also examined the bibliographies of publications selected for inclusion in our review for other relevant studies. PROSPERO registration number: CRD42020148742.

Results: We identified 1,264 original clinical trials conducted between 1974 and 2020, in many countries around the world, enlisting men and women from almost all ethnicities, backgrounds, age groups and with different comorbidity burdens. One trial reported a single ALF case with gemifloxacin and the other reported another case with moxifloxacin.

Conclusion: There is inadequate evidence from clinical trials to implicate quinolone antibiotics as a cause of acute liver failure.

\section{Keywords}

Acute liver failure, Clinical trials, Drug safety, Pharmacovigilance, Quinolones, Systematic review

\section{Introduction}

Quinolones are synthetic antibiotics that continue to gain popularity on a global scale since they were introduced in the mid-1960s (Table 1) [1]. The popularity of quinolones may be attributed to their potency, broad spectrum, unique mechanism of action and strong pharmacologic profile relative to other antibiotic classes [2-8]. Despite the development of adverse events, emergence of resistance, and the availability of other treatment alternatives [2], this favored status persisted. Whereas many researchers consider

Citation: Taher MK, Habsah M, Bjerre L, Momoli F, Mattison D, et al. (2021) Systemic Quinolones and Risk of Acute Liver Failure II: Systematic Review of Clinical Trials. Clin Med Rev Case Rep 8:361. doi. org/10.23937/2378-3656/1410361

Accepted: August 28, 2021: Published: August 30, 2021

Copyright: (c) 2021 Taher MK, et al. This is an open-access article distributed under the terms of the Creative Commons Attribution License, which permits unrestricted use, distribution, and reproduction in any medium, provided the original author and source are credited. 
Table 1: Four generations of quinolone antibotics.

\begin{tabular}{|l|l|}
\hline Generation & Quinolone Antibiotic \\
\hline First & Nalidixic acid, cinoxacin, flumequine, oxolinic acid, piromidic acid, pipemidic acid, rosoxacin \\
\hline Second & Lomefloxacin, norfloxacin, ciprofloxacin, ofloxacin, fleroxacin, pefloxacin, rufloxacin \\
\hline Third & Levofloxacin, sparfloxacin, temafloxacin, grepafloxacin, balofloxacin, pazufloxacin, tosufloxacin \\
\hline Fourth & $\begin{array}{l}\text { Moxifloxacin, gemifloxacin, trovafloxacin, gatifloxacin, clinafloxacin, garenoxacin, sitafloxacin, prulifloxacin, } \\
\text { finafloxacin }\end{array}$ \\
\hline
\end{tabular}

quinolones as having a reasonable safety profile, with most adverse reactions being mild to moderate and self-limiting, some quinolones have been withdrawn from the market and others have sustained restricted usage advisories [8-11].

Acute liver failure (ALF) is a serious disease involving rapid, progressive and severe loss of hepatic cells without evidence of current or previous liver impairment [1214]. While this loss is sometimes reversible, it is more likely to progress rapidly to patient death unless liver transplantation is carried out in a timely manner [1214]. This loss of hepatic cell occurs within 4-6 weeks of a triggering factor [15-17]. These factors including viruses, bacteria, toxins, drugs, and herbal and nutritional supplements [12-14,18-23]. In the US, a recent study reported ALF incidence between 1-6 per million every year, in addition to encompassing $7 \%$ of liver-related transplants and $6 \%$ of liver-related deaths [15].

Drug induced liver injury (DILI) has been repeatedly reported as the most common cause of ALF in Europe and United States, while also representing the most common cause for drug withdrawal by regulatory agencies due to its life-threatening repercussions $[12,13,18,20,21,24-26]$.

DILI is a diagnosis of exclusion and depends, due to absence of a credible gold standard, on physician judgment, careful medical and medication histories, and exclusion of all other possible risk factors [20-22,2628]. In 2014, the American College of Gastroenterology released their revised criteria for diagnosing DILI, with the hope of establishing a gold standard test for diagnosing this condition [29].

In the absence of solid evidence from clinical trials and pharmacoepidemiology studies, regulators such as FDA and EMA often use spontaneous adverse event and case reports to guide their safety-based responses, irrespective of the existence of solid evidence of causality [2,9,30-41].

This review represents the second of three complementary studies that examines the association of systemically administered quinolone antibiotics with ALF risk, which focuses on results from clinical trials. The first part provides an in-depth analysis of ALF reports associated with quinolones, which had been recorded in the US FDA adverse event reporting system (FAERS) between 2010-2019 [42]. The third part involves a nested case-control study based on a major US electronic health records database that includes detailed medical and sociodemographic information on more than 70 million patients from 500 different hospitals across the US between 2000-2016 [43].

\section{Methods}

\section{Review strategy}

We implemented a comprehensive, multi-step search strategy to identify original studies that examined the association between quinolone antibiotics with the risk of acute liver failure. The search was conducted in accordance with the PRISMA guidelines (Preferred Reporting Items for Systematic Reviews and MetaAnalyses), and following the specific guidance provided by the Cochrane Collaboration [44].

The search strategy was designed and implemented between April 16-22, 2017, and updated on April 1-3, 2020 to identify all relevant original studies related to each of the 30 members of the quinolone class of antibiotics shown in Table 1.

We searched multiple bibliographic databases, clinical trial registries and major grey literature sources such as drug review networks and databases of pharmaceutical companies, relevant national and international agencies and international conference proceedings. Experts were consulted on all components of the review strategy and implementation. We also inspected the bibliographies of examined studies for additional relevant studies not already identified via the original search.

This review has been registered in the international prospective register of systematic reviews PROSPERO under reference number CRD42020148742.

\section{Criteria for considering studies for this review}

This review focuses on the safety rather than efficacy of quinolone antibiotics. The outcome measure of interest is the number of cases with a de-novo diagnosis of acute liver failure within 30 days of systemic administration of a quinolone antibiotic in an otherwise liver disease-free individual.

Eligibility criteria for inclusion in this review comprise original clinical trials examining a single systemicallyadministered quinolone antibiotic compared to a placebo, another non-quinolone antibiotic or non- 
quinolone-containing antibiotic combination. Trials with multiple arms, which include a single quinolone on one arm and a placebo on one of the other arms, would be considered eligible.

Trials where participants had prior or existing liver impairment, trials testing topical/non-systemically administered quinolones, a combination of quinolones, or a combination of quinolones and other drugs, and trials testing efficacy of different doses of a quinolone antibiotic, or testing a quinolone against another quinolone were excluded from this review. Cross-over trials were also excluded to avoid any possible carryover effect from the initial intervention. Non-original studies, observational studies and trials where no results were available were also excluded. We enforced no restrictions on participants' age, sex, racial/ethnicity or any other background characteristics.

\section{Search methods for identification of studies}

Bibliographic databases searched included Medline (Ovid MEDLINE ${ }^{\circledR}$ In-Process \& Other Non-Indexed Citations and Ovid MEDLINE ${ }^{\circledR} 1946$ to Present), EMBASE (Embase Classic + Embase 1947 to 2017 May 02), Cochrane Central Register of Controlled Trials (CENTRAL), and CINAHL (Cumulative Index to Nursing and Allied Health Literature).

Search terms included medical subject headings and text word search for quinolone antibiotics both as a class and as individual agents, in addition to acute liver failure as the outcome of interest. The search integrated a specialized search algorithm with reported high efficiency for identifying clinical trials in MEDLINE Ovid, EMBASE and CINAHL [45]. We also used another specialized algorithm for identifying adverse reactions [46] with the MEDLINE and EMBASE databases only. No language, time or other filters were used to limit the search output. A detailed description of the used search terms is given in Supplementary Material I. Complete listings of included studies, excluded studies and reason for exclusion are given in Supplementary Material II, III \& IV respectively.

A more extensive search was done for all clinical trials conducted on any of the quinolone antibiotics documented in any of the major international clinical trial registries, including the World Health Organization Clinical Trials Registry (ICTRN) and United States Clinical Trials Database (Clinicaltrials.gov), as well as similar registries from the European Union, United Kingdom, Netherlands, Japan, China, South Korea, India, Iran, Australia and South Africa. We also searched major grey literature sources including international conference proceedings, drug review networks and databases of pharmaceutical companies for ongoing or unpublished studies. Some relevant background publications were used to support this review process.
Duplicate screening of titles and abstracts (Level 1) and full-text examination (Level 2) were performed independently by two reviewers ( $\mathrm{MT}$ and $\mathrm{MH}$ ) to identify studies eligible for inclusion in the review, based on the predetermined eligibility criteria. Conflicts identified in each step were resolved via consensus between the reviewers or via a panel of senior scientists (FM, LB, DM, DK), prior to moving to the next step.

In the event where multiple publications report on an original study/trial, only the primary publication/ record was assessed in the review. All studies which reported on, or have been indexed to a specific original study/trial, were listed under the original study/trial record. Maximum diligence was adopted to include all papers reporting on each of the included clinical trials. A complete listing of original studies and their reporting/ indexed publications is included in Supplementary Material IV.

\section{Data collection and analysis}

We collated the references identified from all sources using EndNote [47] reference management application version 8.2, which was used to flag potential duplicates, with manual resolution employed to remove actual duplicates. We used Review Manager application version 5.3 [48] to collate and classify all examined studies, tabulate the reasons for study exclusion, and compile the information required for generating the PRISMA flow diagram [49].

Data abstraction spreadsheets were developed using Microsoft Office Excel, and used to abstract the following information study (design, year, country), tested quinolone antibiotic (name, dose, route of administration, frequency, duration), control agent/ group (type, name, dose, route of administration, frequency, duration), participant characteristics (total sample size, age, sex, race), comorbidities (prior liver conditions, diabetes, alcohol abuse), treatment period and follow-up (average, range, years), main result(s), and authors' reported conclusion. Reviewers' comments, if any, are also included.

\section{Results}

\section{Flow of studies}

The search strategy resulted in retrieval of 3,560 records, including 3,521 records from bibliographic databases and clinical trial registries, 30 records from the databases of pharmaceutical companies, and 1 record from an international conference. Electronic and manual de-duplication resulted in removal of 774 records. An additional 260 non-quinolone-related studies were excluded, leaving 2,526 studies for fulltext examination. Examined studies spanned across 5 decades (1974-2020), peaking between 2008-2012 (Figure 1). These studies were conducted in many countries around the world, enlisting participants from 


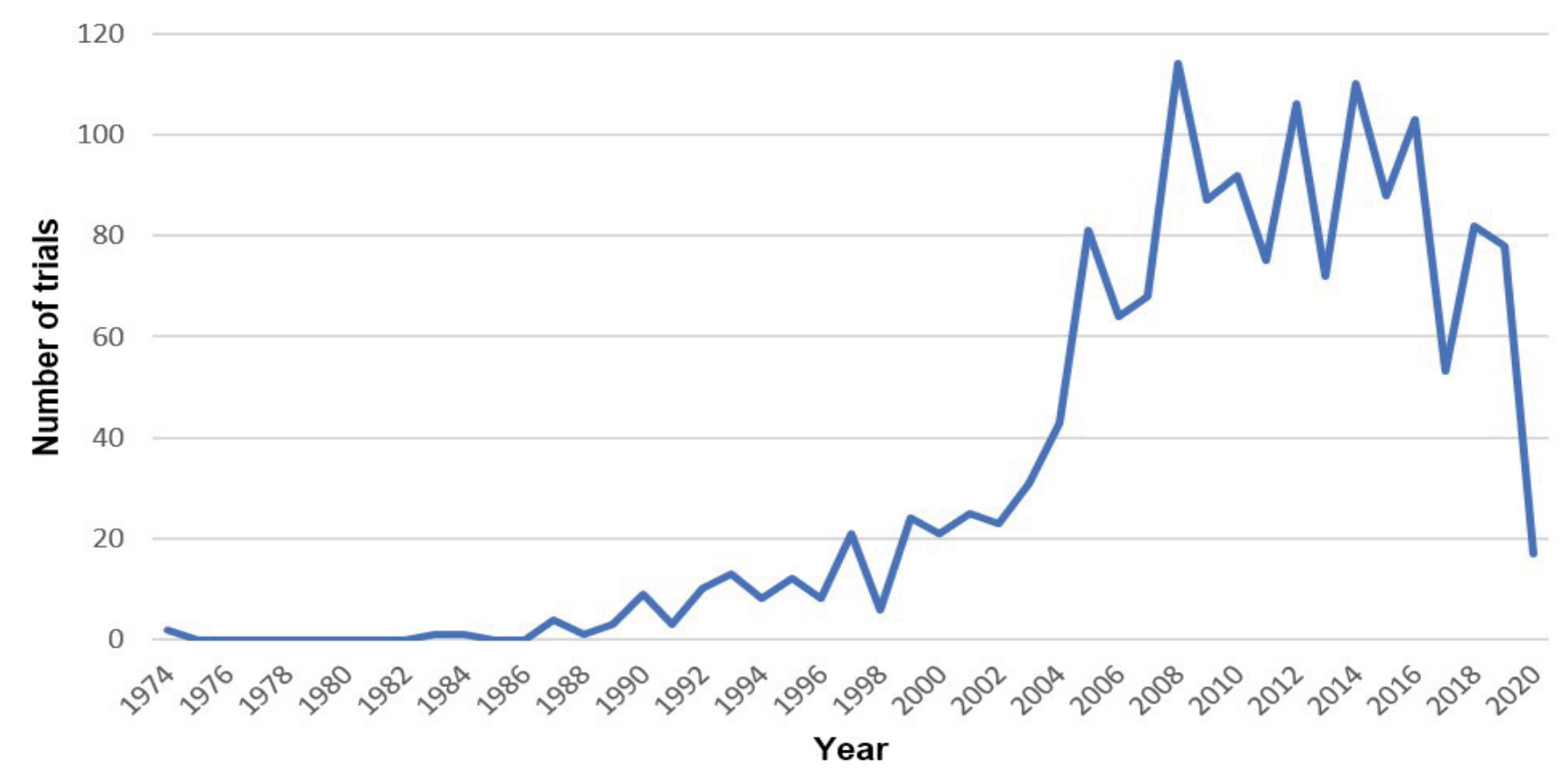

Figure 1: Temporal distribution of clinical trials.

Table 2: Reasons for study exclusion.

\begin{tabular}{|c|c|c|}
\hline Exclusion group & Reason for exclusion & \# Of References \\
\hline Duplicate references (774) & Duplicate studies & 774 \\
\hline Irrelevant exposure (260) & Non-quinolone related studies & 260 \\
\hline Unavailable full-text (6) & Reference could not be retrieved & 6 \\
\hline Irrelevant population (41) & Current or prior liver impairment & 41 \\
\hline Non-original studies (971) & Non-original studies & 971 \\
\hline \multirow[t]{8}{*}{ Irrelevant study design $(1,190)$} & Quinolone combined with other drugs & 284 \\
\hline & Cross-over trial & 194 \\
\hline & No results available & 167 \\
\hline & Trial with multiple arms & 149 \\
\hline & Quinolone in a topical formulation & 126 \\
\hline & Observational study & 106 \\
\hline & Trial with a single arm & 96 \\
\hline & Quinolones on multiple arms & 68 \\
\hline \multirow[t]{6}{*}{ Irrelevant publication type (179) } & Review article & 89 \\
\hline & Case report/case series & 73 \\
\hline & Letter/commentary & 7 \\
\hline & Not a clinical trial & 6 \\
\hline & Report & 3 \\
\hline & Conference abstract & 1 \\
\hline Eligible trials & $\begin{array}{l}\text { Eligible clinical trials with no reporting on acute } \\
\text { liver failure }\end{array}$ & 137 \\
\hline
\end{tabular}

all sexes, ethnicities, age groups and with different types and levels of comorbidities.

Among 1,555 original quinolone-related studies identified from our screening and examination of retrieved evidence; we were able to identify 138 trials that met the eligibility criteria for inclusion. Only two of these 138 trials $[50,51]$ reported on the incidence of acute liver failure in participants who received a systemically administered mono-quinolone antibiotic, with no history of apparent, current or prior liver conditions/diseases. Accordingly, we were unable to retain enough number of studies to conduct a quantitative analysis. A summary of the reasons for exclusion is provided in Table 2, with more details given in a Supplementary Materials III \& IV. A detailed PRISMA flow diagram [49] is shown in Figure 2. 


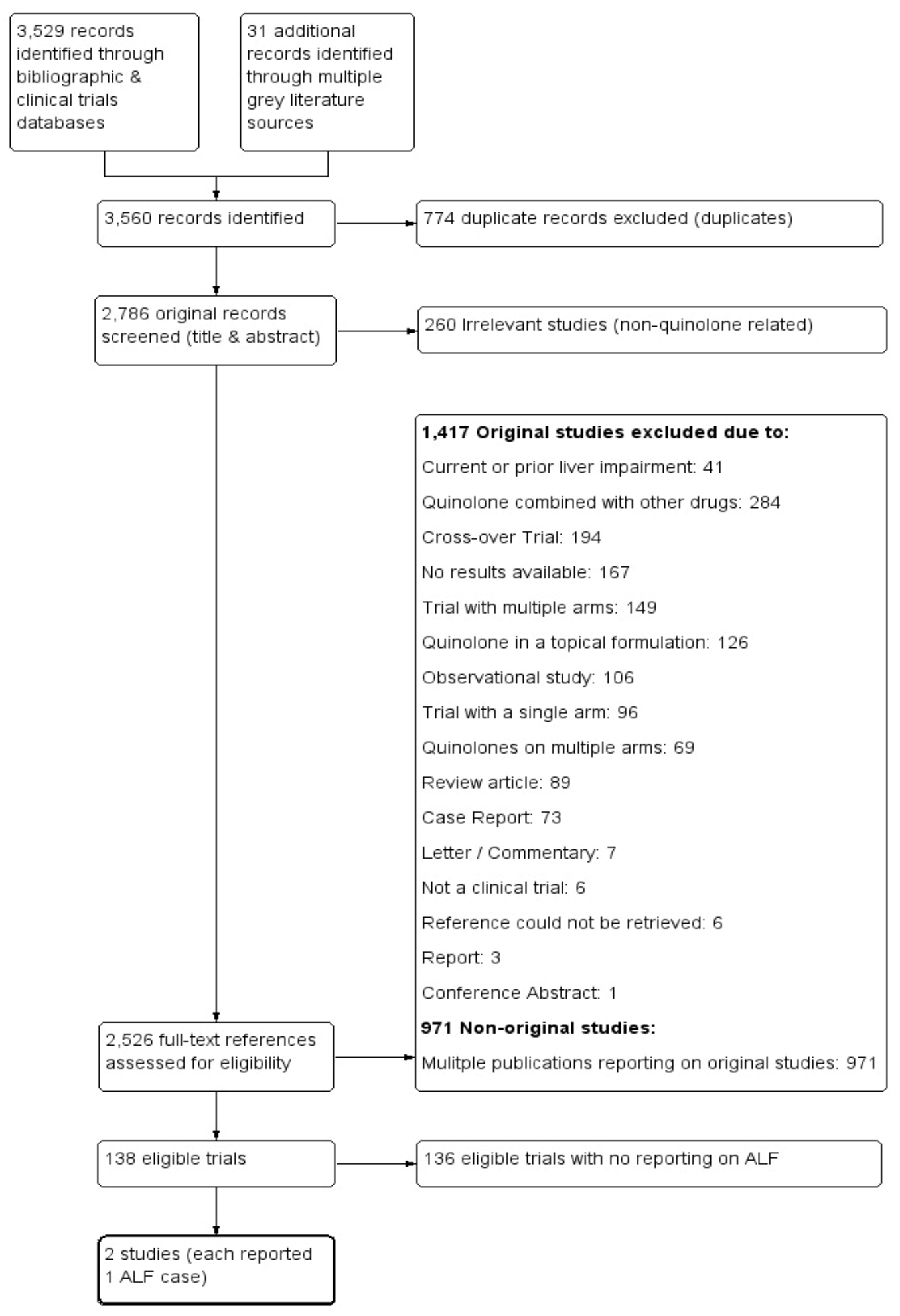

Figure 2: PRISMA flow diagram.

\section{Characteristics of included studies}

In a 2004 multicenter, randomized, double-blinded trial, 1 case of hepatocellular damage was reported in the gemifloxacin group compared to 2 cases in the amoxicillin/clavulanate (control) group [50]. Another 2007 prospective, randomized, double-dummy, doubleblind, multicenter, non-inferiority trial was conducted in 52 centers in Argentina, Belgium, Bulgaria, Estonia, France, Germany, Greece, Israel, Latvia, Lithuania, Romania, Russia, South Africa, and Spain [51]. This study reported one case of acute liver failure in the moxifloxacin group, compared to none in the ertapenem group. Characteristics of included studies are summarized in Table 3. Further details on these studies, including assessment of quality of included studies using the Cochrane risk of bias tool [52], are included in Supplementary Material II.

\section{Summary of excluded studies}

The great majority all the examined studies $(2,388$ out of 2,526 ) were excluded from any further qualitative 


\begin{tabular}{|c|c|}
\hline 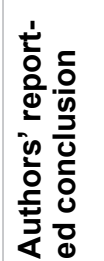 & 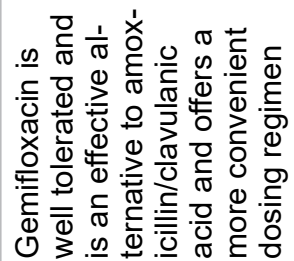 \\
\hline
\end{tabular}

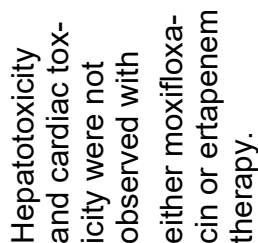

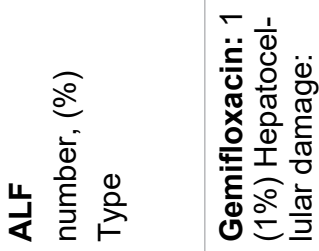

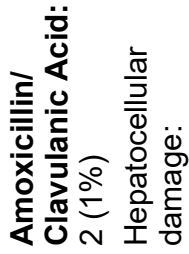

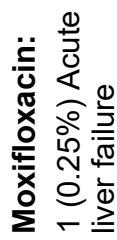

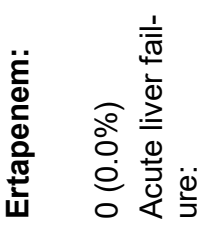

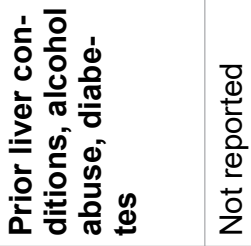

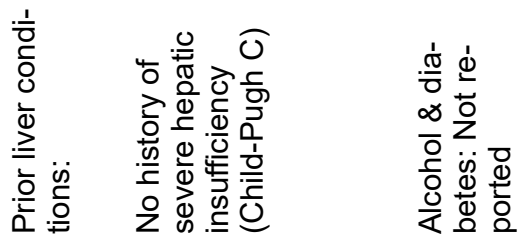
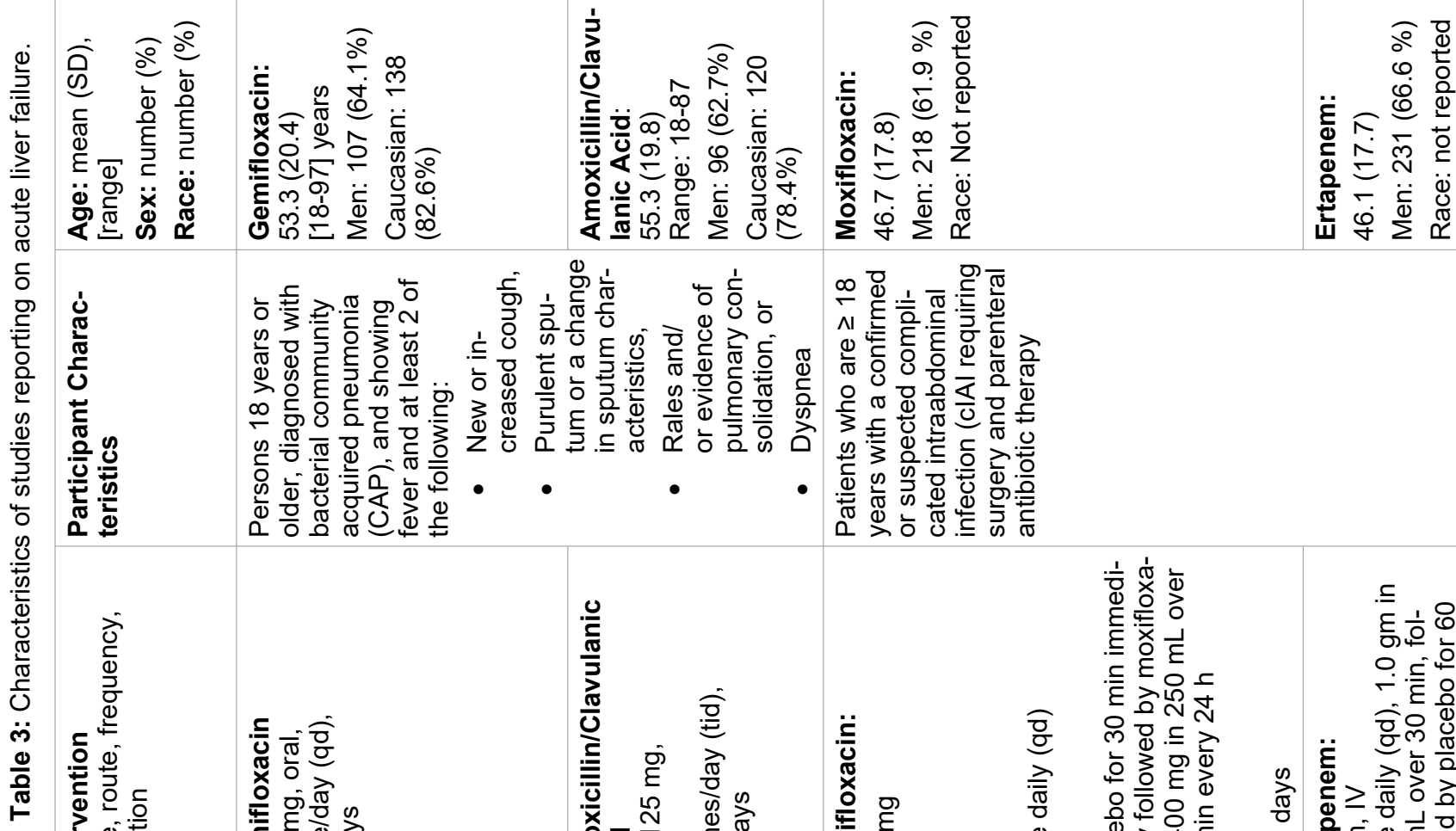

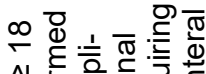
政
光
记

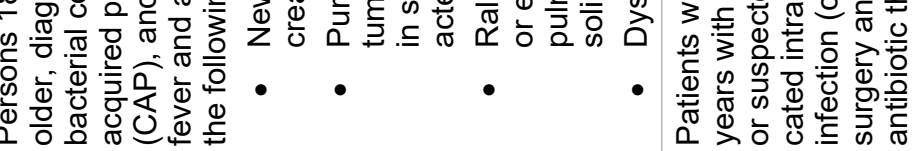

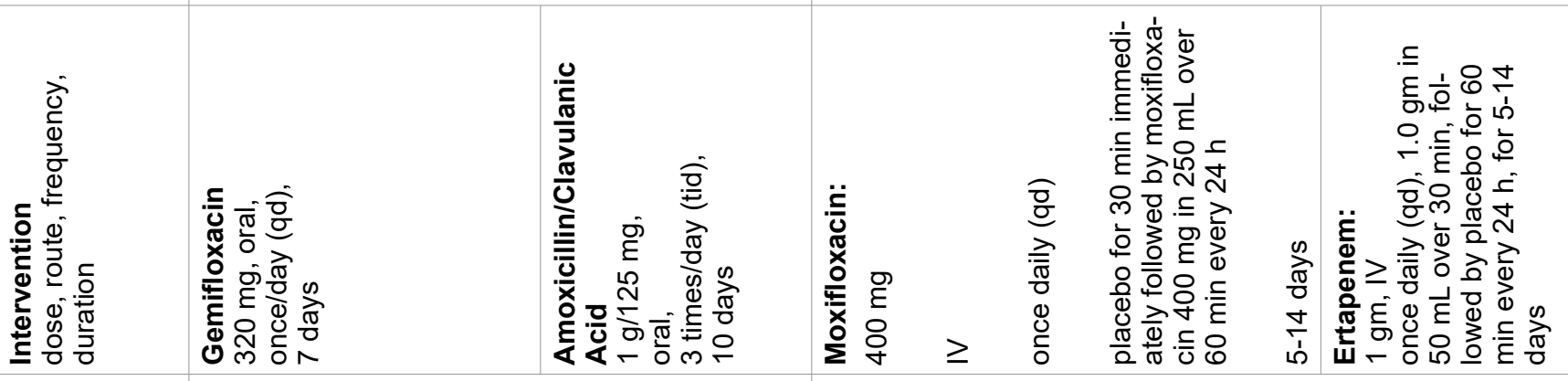

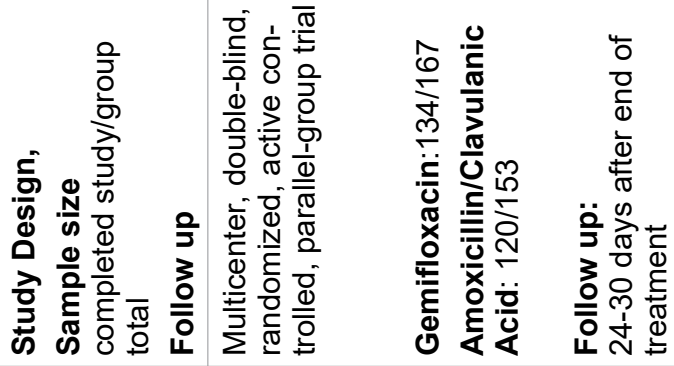
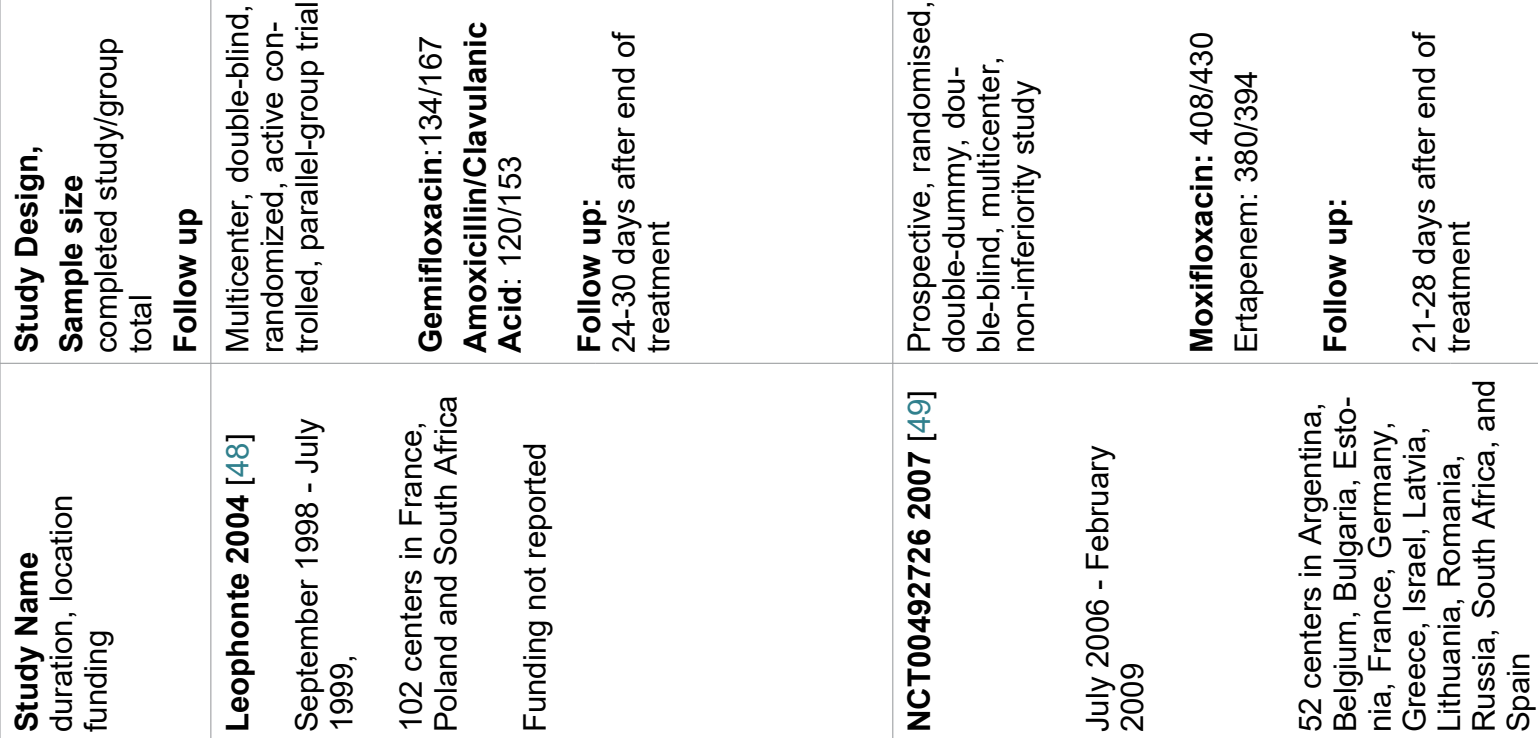
or quantitative analysis based on the predetermined inclusion criteria. Whenever a trial had more than one reason for exclusion, we reported on the most compelling reason. A summary list of the reasons for exclusion of these trials is shown in Table 2. A detailed listing of the full citations of all excluded studies is given in Supplementary Material III. There were 136 other eligible trials with no reporting on the occurrence of ALF in the systemic mono-quinolone group arm. Results from an additional 167 trials were not available at the time of completion of this review.

\section{Discussion}

\section{Interpretation of findings}

Acute liver failure is a serious adverse drug reaction that develops quickly, signaling rapid deterioration of liver function, and requiring immediate medical attention, predominantly, if not exclusively, in hospital and/or emergency room settings. Although ALF may resolve in some cases, this potentially fatal condition is often severe enough to mandate timely liver transplantation.

In the 138 eligible trials, there were only two original clinical trials that each reported a single case of acute liver failure, one with Gemifloxacin [50] and one with moxifloxacin [51]. This result constitutes insufficient evidence to implicate systemically administered quinolone antibiotics as being associated with acute liver failure. Neither of these two trials $[50,51]$ suggested that the occurrence of ALF was attributable to treatment with quinolones; a publication [53] reporting on one of the two trials [51] specifically reported "no evidence of any treatment effect on the incidence of hepatic events".

As we were unable to identify more than one clinical trial for each of gemifloxacin and moxifloxacin, a meta-analysis was not possible. Using the Cochrane risk of bias tool [52], both trials showed a low risk of bias. However, an overall assessment of the identified evidence using the GRADE framework [54] was also not feasible since we were unable to assess certainty of evidence (inconsistency, indirectness, imprecision, publication bias, large effect, plausible confounding, dose-response gradient), which require multiple studies to generate meaningful outcomes.

Our review was focused on identifying evidence from clinical trials regarding de novo development of quinolone-induced ALF, in persons with an otherwise healthy liver. However, we identified three eligible trials that each reported a single case of either hepatic failure (not ALF) with the quinolone comparator [55,56], or hepatic cirrhosis with ciprofloxacin (unrelated to treatment) [57]. A fourth uncontrolled trial reported a case of liver dysfunction in the moxifloxacin group on the fifth day of treatment. Despite discontinuation of moxifloxacin, the patient did not sustain recurrence of pneumonia symptoms or require further antibiotic treatment.

To the best of our knowledge, this is the first systematic review that examined all original studies reporting on clinical trials that tested any of the quinolone antibiotics, in relation to their association with the risk of acute liver failure. This review covers all publicly available original, peer-reviewed publications, as well as other grey literature sources for unpublished clinical trials conducted with quinolone antibiotics. Clinical trials represent the most rigorous assessment of human drug safety, that is used to examine the association between a specific $\operatorname{drug}(\mathrm{s})$ and specific adverse reactions (ADR) [34,58].

However, clinical trials may be subject to a lack of representativeness since the characteristics of the selected participants will not mirror all possible demographics, risk factors, and underlying determinants in the general population who will be using the approved drug under real-life situations [34,38,58-60]. By design, clinical trials might miss rare ADR such as ALF due to the limited number of participants [34,38,58,61,62].

\section{Conclusion}

As acute liver failure (ALF) is a serious medical emergency that requires immediate medical attention, cases with suspected diagnosis cannot be handled in physician offices and must be immediately admitted to a hospital for further assessment and/or intervention. Although it might not have been one of the outcomes of interest in a clinical trial, any occurrence of ALF in a trial participant cannot go unnoticed. In the event of a case of ALF, treatment must be stopped and the patient managed immediately, while reporting the incident to the relevant regulatory authorities. The unequivocal lack of reporting on incidence of quinolone-related ALF by almost all trials from 1974 to date, across different jurisdictions, enlisting participants from both sexes, encompassing almost all ethnicities, backgrounds, age groups and comorbidity profiles, leaves little room for any undetected/unreported occurrences of quinoloneassociated ALF.

The absence of an abundance of cases of ALF in the clinical trials included in the present review, while reassuring, does not rule out the possibility of an increased risk in the general public under real world conditions of use. Observational Pharmacovigilance studies based on large and diverse patient populations are needed to confidently confirm or reject an association between use of quinolones and increased risk of acute liver failure.

\section{Author Contributions}

The primary author, Mohamed Taher, designed and implemented the review strategy including search methodology, design of study screening and data 
abstraction forms, reference collation and full-text acquisition, screening and examination of the identified references, data abstraction and drafting of this manuscript. Mohamed Habsah independently assessed all studies examined in this review. Lise Bjerre, Franco Momoli, Donald Mattison and Daniel Krewski provided guidance and feedback on all aspects of the review design and implementation, as well as critical review and approval of the manuscript.

\section{Source of Funding}

This research was supported in part with funding from the McLaughlin Centre for Population Health Risk Assessment at the University of Ottawa. D. Krewski is the Natural Sciences and Engineering Research Council of Canada Chair in Risk Science at the University of Ottawa.

\section{Declaration of Interest}

All authors who contributed to both this study and manuscript report no conflict of interest in relation to the planning for and conducting this study as well as production of this manuscript.

\section{References}

1. Committee on Infectious Diseases (2006) The use of systemic fluoroquinolones. Pediatrics 118: 1287-1292.

2. Liu $H(2010)$ Safety profile of the fluoroquinolones: Focus on Levofloxacin. Drug Saf 33: 353-369.

3. Transparency Market Research (2014) Antibacterial drugs market expected to reach USD 45.09 billion globally in 2019: Transparency market research.

4. Appelbaum P, Hunter P (2000) The fluoroquinolone antibacterials: Past, present and future perspectives. Int $J$ Antimicrob Agents 16: 5-15.

5. Emmerson A, Jones A (2003) The quinolones: Decades of development and use. J Antimicrob Chemother 51(Suppl. S1): $13-20$.

6. Furiex (2012) Novel fluoroquinolone (JNJ-Q2).

7. Heeb S, Fletcher M, Chhabra S, Diggle S, Williams $P$, et al. (2011) Quinolones: From antibiotics to autoinducers. FEMS Microbiol Rev 35: 247-274.

8. Bolon M (2011) The newer fluoroquinolones. Med Clin North Am 95: 793-817, viii.

9. Lode H (2010) Safety and tolerability of commonly prescribed oral antibiotics for the treatment of respiratory tract infections. Am J Med 123: S26-S38.

10. Cuzzolin L, Fanos V (2002) Safety of fluoroquinolones in paediatrics. Expert Opin Drug Saf 1: 319-324.

11. Stahlmann R, Lode $H$ (2013) Risks associated with the therapeutic use of fluoroquinolones. Expert Opin Drug Saf 12: $497-505$.

12. Gulen M, Ay MO, Avci A, Acikalin A, Icme F (2015) Levofloxacin-induced hepatotoxicity and death. Am J Ther 22: e93-e96.

13. Lee WM (2013) Drug-induced acute liver failure. Clin Liver Dis 17: $575-586$, viii.

14. Orman ES, Conjeevaram HS, Vuppalanchi R, Freston JW,
Rochon J, et al. (2011) Clinical and histopathologic features of fluoroquinolone-induced liver injury. Clin Gastroenterol Hepatol 9: 517-523.e3.

15. Montrief T, Koyfman A, Long B (2019) Acute liver failure: A review for emergency physicians. Am J Emerg Med 37: 329-337.

16. O'Grady JG, Schalm SW, Williams R (1993) Acute liver failure: Redefining the syndromes. Lancet 342: 273-275.

17. Bernal W, Wendon J (2013) Acute liver failure. N Engl J Med 369: 2525-2534.

18. Radovanovic M, Dushenkovska T, Cvorovic I, Radovanovic N, Ramasamy $V$, et al. (2018) Idiosyncratic drug-induced liver injury due to ciprofloxacin: A report of two cases and review of the literature. Am J Case Rep 19: 1152-1161.

19. Leitner JM, Graninger W, Thalhammer F (2010) Hepatotoxicity of antibacterials: Pathomechanisms and clinical. Infection 38: 3-11.

20. Babai S, Auclert L, Le-Louet H (2018) Safety data and withdrawal of hepatotoxic drugs. Therapie.

21. Katarey D, Verma S (2016) Drug-induced liver injury. Clin Med (Lond) 16: s104-s109.

22. Leise MD, Poterucha JJ, Talwalkar JA (2014) Drug-induced liver injury. Mayo Clin Proc 89: 95-106.

23. Raschi E, Poluzzi E, Koci A, Salvo F, Pariente A, et al. (2015) Liver injury with novel oral anticoagulants: Assessing postmarketing reports in the US food and drug administration adverse event reporting system. $\mathrm{Br} \mathrm{J}$ Clin Pharmacol 80: 285-293.

24. (2011) National Institute of Diabetes and Digestive and Kidney Diseases-Bethesda (MD). Drug-Induced Liver Injury - Overview. NIH-DILIN.

25. Chalasani N, Fontana RJ, Bonkovsky HL, Watkins PB, Davern T, et al. (2008) Causes, clinical features, and outcomes from a prospective study of drug-induced liver injury in the United States. Gastroenterology 135: 19241934.

26. Bissell DM, Gores GJ, Laskin DL, Hoofnagle JH (2001) Drug-induced liver injury: Mechanisms and test systems. Hepatology 33: 1009-1013.

27. Ghabril M, Chalasani N, Bjornsson E (2010) Drug-induced liver injury: A clinical update. Curr Opin Gastroenterol 26: 222-226.

28. Licata A (2016) Adverse drug reactions and organ damage: The liver. Eur J Intern Med 28: 9-16.

29. Chalasani NP, Hayashi PH, Bonkovsky HL, Navarro VJ, Lee WM, et al. (2014) ACG Clinical Guideline: The diagnosis and management of idiosyncratic drug-induced liver injury. Am J Gastroenterol 109: 950-966.

30. Owens R (2004) QT prolongation with antimicrobial agents: Understanding the significance. Drugs 64: 1091-1124.

31. Harpaz R, Vilar S, Dumouchel W, Salmasian H, Haerian $\mathrm{K}$, et al. (2013) Combing signals from spontaneous reports and electronic health records for detection of adverse drug reactions. J Am Med Inform Assoc 20: 413-419.

32. Huang Y, Moon J, Segal J (2014) A comparison of active adverse event surveillance systems worldwide. Drug Saf 37: 581-596.

33. Moore N (2013) The past, present and perhaps future of pharmacovigilance: Homage to Folke Sjoqvist. Eur J Clin Pharmacol 69: 33-41. 
34. Harmark L, van Grootheest A (2008) Pharmacovigilance: Methods, recent developments and future perspectives. Eur J Clin Pharmacol 64: 743-752.

35. Pal S, Duncombe C, Falzon D, Olsson S (2013) WHO strategy for collecting safety data in public health programmes: Complementing spontaneous reporting systems. Drug Saf 36: 75-81.

36. World Health Organization (2007) A practical handbook on the pharmacovigilance of antimalarial medicines.

37. World Health Organization (2012) A practical handbook on the pharmacovigilance of medicines used in the treatment of tuberculosis: Enhancing the safety of the TB patient.

38. Waller $P$ (2010) An introduction to pharmacovigilance. (2 edn), Wiley-Blackwell, Chichester, West Sussex, UK.

39. Honig P (2013) Advancing the science of pharmacovigilance. Clin Pharmacol Ther 93: 474-475.

40. (2008) Institute of Medicine. Pharmacovigilance. In: Emerging Safety Science: Workshop Summary. National Academy of Sciences, Washington DC.

41. La Rochelle P, Lexchin J, Simonyan D (2016) Analysis of the drugs withdrawn from the us market from 1976 to 2010 for safety reasons. Pharmaceutical Medicine 30: 277-289.

42. Taher MK, Alami A, Gravel CA, Tsui D, Bjerre LM, et al. (2021) Systemic quinolones and risk of acute liver failure I: Analysis of data from the US FDA adverse event reporting system. JGH Open 5: 778-784.

43. Taher MK, Crispo JAG, Fortin Y, Moog R, McNair D, et al. (2021) Systemic quinolones and risk of acute liver failure III: A nested case-control study using a US electronic health records database. J Gastroenterol Hepatol 36: 2307-2314.

44. Higgins JPT, Green S (2011) Cochrane Handbook for Systematic Reviews of Interventions. Cochrane Collaboration, Version 5.1.0.

45. Scottish Intercollegiate Guidelines Network (2014) SIGN Search Filters Guidelines.

46. Golder S, Loke YK (2012) Sensitivity and precision of adverse effects search filters in MEDLINE and EMBASE: A case study of fractures with thiazolidinediones. Health Info Libr J 29: 28-38.

47. (2018) Endnote. Philadelphia, PA, USA.

48. (2014) The Nordic Cochrane Centre, The Cochrane Collaboration, Copenhagen. Version 5.3.

49. Moher D, Liberati A, Tetzlaff J, Altman DG (2009) Preferred reporting items for systematic reviews and meta-analyses: The PRISMA statement. PLoS Med 6: e1000097.

50. Leophonte P, File T, Feldman C (2004) Gemifloxacin once daily for 7 days compared to amoxicillin/clavulanic acid thrice daily for 10 days for the treatment of communityacquired pneumonia of suspected pneumococcal origin. Respir Med 98: 708-720.
51. NCT00492726 (2007) A prospective, randomized, doubledummy, double-blind, multicenter trial comparing the safety and efficacy of moxifloxacin $400 \mathrm{mg}$ IV QD 24 hours to that of ertapenem $1.0 \mathrm{~g}$ IV QD 24 hours for 5 to 14 days for the treatment of subjects with complicated intra-abdominal infections (PROMISE study). In: Therapy of Complicated Intra-Abdominal Infections With Moxifloxacin or Ertapenem.

52. Higgins JPT, Altman DG, Gotzsche PC, Juni P, Moher D, et al. (2011) The Cochrane Collaboration's tool for assessing risk of bias in randomised trials. BMJ 343: d5928.

53. De Waele JJ, Tellado JM, Alder J, Reimnitz P, Jensen M, et al. (2013) Randomised clinical trial of moxifloxacin versus ertapenem in complicated intra-abdominal infections: Results of the PROMISE study. Int J Antimicrob Agents 41: 57-64.

54. Schünemann H, Brożek J, Guyatt G, Oxman A (2013) Handbook for grading the quality of evidence and the strength of recommendations using the GRADE approach.

55. NCT02531438 (2015) A Phase 3 Randomized, DoubleBlind, Multi-Center Study to Compare the Safety and Efficacy of Omadacycline Intravenous (IV)/Oral (PO) to Moxifloxacin IV/PO for Treating Adults Subjects With Community-Acquired Bacterial Pneumonia. In: Omadacycline vs Moxifloxacin for the Treatment of CABP (EudraCT \#2013-004071-13).

56. EUCTR2013-004071-13-SK (2013) Study to compare the safety and efficacy of Omadacycline versus Moxifloxacin for adults subjects with pneumonia acquired infectiously from normal social contact. In: Paratek Pharma LLC, trans.

57. EUCTR2013-005366-19-HU (2013) A Multicenter, Randomized, Double-Blind, Placebo-Controlled Study to Evaluate the Safety and Efficacy of Pulmaquin ${ }^{\circledR}$ (ARD3150, Dual Release Ciprofloxacin for Inhalation) in subjects who have a lung infection that includes the bacteria Pseudomonas aeruginosa due to non-cystic fibrosis bronchiectasis. This study includes a 28-day open-label extension (all patients will receive study drug for the last 28 days of the study). In: Aradigm C, trans.

58. Coloma PM, Trifiro G, Patadia V, Sturkenboom M (2013) Postmarketing safety surveillance: Where does signal detection using electronic healthcare records fit into the big picture? Drug Saf 36: 183-197.

59. Scurti V, Romero M, Tognoni G (2012) A plea for a more epidemiological and patient-oriented pharmacovigilance. Eur J Clin Pharmacol 68: 11-19.

60. Friedman LM, Furberg CD, DeMets DL, Reboussin DM, Granger CB (2015) Fundamentals of clinical trials. ( $5^{\text {th }}$ edn), Springer, New York.

61. Moses C, Celi LA, Marshall J (2013) Pharmacovigilance: An active surveillance system to proactively identify risks for adverse events. Popul Health Manag 16: 147-149.

62. FDA, CDER and CBER (2005) Guidance for Industry: Good Pharmacovigilance Practices and Pharmacoepidemiologic Assessment. 